TRANS · núm. $24 \cdot 2020$

MISCELÁNEA · 401-418
Partiendo de la hipótesis de que las lenguas que presentan flexiones de género y número son más permeables a los calcos morfológicos, este estudio prueba la existencia de diferencias flexivas en el lenguaje médico y las interferencias que provoca el trasvase lingüístico entre lenguas afines. Para ello, se compiló un corpus de prospectos en francés (80) y español (80) dado que este género textual asegura una representatividad de la muestra con una cantidad de textos menor. Los resultados muestran variaciones de género y número en terminología presente en este género textual que generan interferencias en la traducción, lo que da como resultado estructuras poco idiomáticas o, por el contrario, poco empleadas en el prospecto. Los resultados también ponen de manifiesto la utilidad del corpus para extraer no solo terminología, sino también contextos de uso especializados para establecer equivalencias adecuadas según el género textual que se traduzca.

PALABRAS CLAVE: prospecto, traducción biosanitaria, variación morfológica, lingüística de corpus, corpus comparable.

\title{
Particularidades morfológicas en traducción biosanitaria del francés al español: el prospecto de medicamentos para uso humano
}

\author{
Manuel Cristóbal Rodrícuez Martínez \\ Universidad Europea del Atlántico
}

Emilio Ortega Arjonilla

Universidad de Málaga

\section{Morphological Features in Medical Translation from French into Spanish: the patient leaflet}

Based on the assumption that flexive languages are permeable to morphological borrowings, this study analyzes the flexive differences of the medical language in French and Spanish. To that aim, we compiled a corpus made out of patient leaflets in French (80) and Spanish (80), given that this text type allows to compile a representative corpus requiring a smaller number of texts. Results showed gender and number variation in the terminology of this text type that would produce several interferences, resulting in structures that do not sound natural in the target language or, on the other hand, that are not used frequently in patient leaflets. Besides, results also highlight the value of corpora to extract not only terminology, but also specialized contexts to provide proper equivalences according to the text type to be translated.

KEY WORDS: patient leaflet, medical translation, morphological variation, corpus linguistics, comparable corpus. 


\section{INTRODUCCIÓN}

Un gran número de investigadores aborda el estudio de la traducción médica desde un punto de vista descriptivo de corte terminológico partiendo del inglés como lengua de partida. Este hecho es un reflejo de la importancia que ha adquirido la combinación lingüística inglés-español para la práctica profesional de la traducción a nivel nacional e internacional. En contraste, hay muchas menos investigaciones en otras combinaciones de idiomas, incluida la combinación lingüística francés-español que, a pesar de todo, y a mucha distancia del inglés, ocupa el segundo puesto en el volumen de textos traducidos al español (Crespo Hidalgo, 2010; Martínez López, 2010a; Sánchez Trigo y Munoa, 2013; Sánchez Trigo y Varela, 2015).

Si bien la investigación en traducción médica del francés se está extendiendo en los últimos años, aún existen carencias en la investigación traductológica aplicada en esta combinación lingüística (francés-español) que exigen llevar a cabo más estudios específicos que den cuenta de las singularidades que encierra la traducción de textos médicos entre estos idiomas. A este respecto, resulta relevante estudiar las variaciones producidas a nivel morfológico en los textos médicos redactados en francés y en español, consecuencia de las asimetrías expresivas y gramaticales existentes entre ambas lenguas.

Esta situación actual en la que el inglés actúa como lingua franca en la comunicación científica a escala internacional hace que sean numerosos los estudios lingüísticos o traductológicos que parten del inglés o se refieren específicamente a la combinación lingüística inglés-español por razones diversas, como las apuntadas más arriba. La situación del francés médico y de la combinación lingüística francés-español es bien distinta y, aunque se han hecho esfuerzos nota- bles en los últimos años por paliar la carencia de investigaciones (Sánchez Trigo y Munoa, 2013; Díaz Alarcón, 2016; Rodríguez Martínez y Ortega Arjonilla, 2018), faltan todavía un gran número de aspectos que requieren atención. Entre estos aspectos resaltan, entre otros, aquellos que son objeto de consideración en este artículo, que versa sobre la variación morfológica y el papel desempeñado por estas variaciones en la construcción léxica originada en los textos médicos en francés y en español, lo que supone una dificultad específica de la traducción médica de esta combinación lingüística.

A este respecto, el presente artículo pretende ser una aproximación a la problemática derivada de las diferencias entre lenguas flexivas en el lenguaje médico entre francés y español mediante un análisis de corpus biosanitario, con el objetivo de extraer casos de variación morfológica en la traducción del ámbito biosanitario. De esta manera, se podrá analizar mediante frecuencias de aparición la relevancia de estos casos en un género textual concreto (el prospecto de medicamentos para uso humano).

Si bien es cierto que el estudio de rasgos sintácticos (Navarro et al., 1997; Mendiluce Cabrera, 2002) y léxicos (Martínez López, 2010a; López Garrido, 2016; Durán-Escribano y ArgüellesÁlvarez, 2017; Rodríguez Ponce, 2017) está presente en la literatura científica, el estudio de la variación morfológica en el discurso médico y su traducción al español no se ha tratado en profundidad en la literatura científica hasta el momento. Por ello, el presente trabajo tiene como objetivo contribuir a la investigación sobre morfología en traducción especializada entre lenguas romances, así como analizar si existe o no cierta permeabilidad en cuanto a las variaciones morfológicas entre lenguas con flexión de género y número mediante la extracción de casos mediante corpus. 


\section{TRADUCCIÓN BIOSANITARIA}

Son numerosos los autores que han propuesto una caracterización de la traducción biosanitaria a partir de dificultades propias del ámbito, características desde un punto de vista traductológico, definición, etc. (Navarro y Hernández, 1997; Ortega Arjonilla, 2003; Martínez López, 2009; Muñoz-Miquel, 2009a y 2014).

Las características más comunes en todas estas propuestas definitorias que ayudan a realizar una aproximación a la traducción biosanitaria se resumen en las que aquí siguen (Rodríguez Martínez, 2017):

- Universalidad. En general, el discurso biosanitario tiende a estar regulado por normas y convenciones, tanto lingüísticas (CIE 10, DSM V) como estilísticas (normas de referencia Vancouver). Esto repercute directamente en una normalización terminológica cuya principal finalidad es la de favorecer un intercambio global de conocimiento científico.

- Estilo objetivo. En esta especialización temática se presupone una ausencia de subjetividad debido al carácter predominante expositivo e informativo del discurso biosanitario. Esto también resulta en una mayor incidencia de lenguaje denotativo, carente de cargas connotativas, característica a la que aspiran la mayoría de los lenguajes de especialidad.

- Contenido verificable. Como se menciona en el apartado anterior, el carácter del discurso biosanitario es expositivo e informativo cuya función principal es divulgar un conocimiento específico. Sin embargo, el conocimiento científico se ha de basar en una justificación (teorías e hipótesis previas, resultados de análisis o experimentos, determinadas leyes universales o científicas, etc.) que permita verificarlo empíricamente.
- Terminología arbitraria. Ausencia de relación entre lengua común y terminología específica del ámbito, lo que origina una terminología críptica (Ortega Arjonilla y Martínez López, 2007) que dificulta la comprensión de personas ajenas al campo de conocimiento en cuestión. Esta característica repercute en la comprensión del texto (tanto original como meta) por parte de un lector lego, lo que obliga al traductor a adquirir conocimientos especializados antes de comenzar la lectura de textos biosanitarios para poder comprender la totalidad del sentido del texto objeto de traducción. Además, una de las características de esta terminología es la presencia no solo de términos crípticos, sino también délficos, lo que dificultaría aún más la identificación del léxico propio de cada ámbito temático y género textual al compartir uso (que no acepciones) con el lenguaje no especializado.

- Coherencia. Característica de gran importancia en el discurso biosanitario, ya que estos textos especializados suelen presentar una estructura lógica y coherente, la cual facilitaría el análisis de la argumentación del texto para verificar los resultados o conclusiones, o incluso la navegación dentro del propio contenido global del texto.

- Lingua franca. La mayoría de los autores se refieren al inglés como la lingua franca de la divulgación científica y de la medicina. Es una lengua imprescindible en la traducción biosanitaria, ya que tiene gran influencia en la divulgación de textos biosanitarios; por otro lado, la comunidad científica estadounidense se considera como la comunidad científica de referencia, por lo que el dominio del inglés permitirá al traductor desenvolverse de manera eficiente entre dicha comunidad incluso en trabajos con combinación lingüística francés-español. 
404

Esto, a su vez, afianza la hegemonía del inglés frente a otras lenguas en la investigación científica.

- Diferentes subámbitos específicos. Aunque el ámbito biosanitario es muy específico, este, a su vez, se divide en multitud de campos con una mayor especificidad, con características y terminología propias, como por ejemplo la fisioterapia, la terapia ocupacional, la ingeniería genética, la oncología, la psiquiatría, la oftalmología, la farmacología, la anatomía, la bioquímica o la cirugía y sus correspondientes especialidades, entre otros. Estos diferentes ámbitos pueden igualmente presentar ciertos rasgos híbridos al compartir campo temático, como es el caso de la ingeniería genética, en la que se pueden encontrar rasgos propios del ámbito biosanitario, así como elementos propios del ámbito tecnológico o industrial.

Estas características no son universales a cada texto de temática biosanitaria, ya que, como se ha mencionado, se trata de un acto comunicativo especializado y cada acto de comunicación posee unos componentes específicos que graduarán la relevancia y la veracidad de estas características propuestas. Así, se encontrarán textos que posean una gran normalización terminológica; otros que posean multitud de fenómenos de polisemia, sinonimia o eponimia $\mathrm{y}$ otros textos que presenten un tenor indirecto y haya que adaptar el discurso científico (terminología críptica, estructura, ausencia de referencias y anglicismos) según las limitaciones diasistemáticas que procedan.

\section{La morfología en la traducción biosanitaria}

El estudio de la morfología en el ámbito de la traducción médica se ha visto en ocasiones limitado a los mecanismos de creación terminológica
(Martínez López, 2008; Eurrutia Cavero, 2017). No obstante, algunos estudios ahondan en sus peculiaridades, los cuales muestran patrones recurrentes y ejemplos de casos representativos en el francés y en el español (Martínez López, 2010a; Rodríguez Martínez, 2017).

A pesar de ello, la literatura científica sobre traducción biosanitaria que contemple el francés como lengua de trabajo no es muy amplio (Rodríguez Martínez, 2017), y con ello se reduce a su vez los estudios sobre la relevancia de la morfología en este par de lenguas con tanta proximidad estructural. Sin embargo, y tal y como se afirma en la literatura científica, la proximidad entre lenguas romances suele provocar «una tendencia a obviar aspectos esenciales cuyo escrupuloso respeto contribuye a que un texto traducido no lo parezca» (Veloso, 2004: 184). De hecho, Veloso (2004) continúa aconsejando que se profundice en estas particularidades y reglas que a priori puedan resultar arbitrarias pero que ayudarían a entender su naturaleza y, consecuentemente, a identificar de manera más eficiente estas variaciones morfológicas que pueden darse en estos contextos.

Estas variaciones morfológicas pueden darse a diferentes niveles, ya que las lenguas flexivas (como el francés y el español) poseen morfemas de género, número, aspecto, modo, etc. (Alarcos Llorach, 2009). Estas particularidades morfológicas se vuelven menos identificables en el proceso traductológico entre el francés y el español, donde se suele pensar que hay cierto nivel de paralelismo estructural (Veloso, 2004) y reproductibilidad (Ortega Arjonilla y Martínez López, 2007). En los contextos especializados, donde prima la objetividad, la precisión y la concisión, la identificación del género suele verse afectada al eliminar los artículos y dejando únicamente la concordancia con elementos circundantes la adscripción de un género u otro, por ejemplo, o 
de su número en elementos léxicos cuyo significante acaba en $/ \mathrm{s} /$ precedida de vocal átona.

Además, no podemos olvidar que las variaciones morfológicas no solo están aludiendo a una diferenciación ligada a un cambio de género en el referente real en el caso del género, o una variación ligada a las cantidades de un elemento en el número. En ciertos casos, una variación de género o número puede suponer un cambio semántico, lo que en contextos especializados supone un problema mayor debido a la precisión y concisión a la que aludíamos anteriormente. Este es el caso del cambio de género en cólera, cuya forma femenina alude a un sentimiento de ira o enfado, mientras que su forma masculina alude a una enfermedad de origen bacteriano. En el caso del cambio de número, nos encontramos, por ejemplo, con pelo, cuya forma en singular alude tanto a un único cabello como al conjunto de ellos, mientras que su forma en plural añade connotaciones de tipo expresivo que no deberían aparecer en textos de carácter científico.

\section{El prospecto farmacéutico como objeto de análisis en traducción}

En la actualidad, el estudio del género textual es una de las líneas de investigación más fructíferas en el ámbito de la lingüística aplicada, más concretamente en el ámbito de la lengua para fines específicos (López, 2000). Este concepto, según López (2000: 99-100) «explica la capacidad que tienen los hablantes de una lengua de reconocer una serie de características y funciones que están prototípicamente presentes en algunos textos y que están asociadas a situaciones sociales habituales de una determinada cultura».

Sin embargo, la diversidad en cuanto a tipologías textuales hace que no suela existir unanimidad en cuanto a la clasificación de un texto.
Así, y dado el caso que nos ocupa, Mayor Serrano 405 (2007: 126) recoge las diferentes catalogaciones del prospecto de medicamentos:

Los investigadores no consiguen ponerse de acuerdo a la hora de «catalogar» determinadas clases de texto. Ilustraré mis palabras con un caso concreto: el prospecto de medicamento. Así, para Gamero Pérez (1999-2000), el prospecto de medicamento es un texto perteneciente al campo técnico, y lo cataloga como texto expositivo con foco secundario instructivo. Muñoz (2002) [...] considera que el prospecto de medicamento es un texto médico, y lo clasifica bajo la función/tono instructivo + expositivo. Por último, Pérez García (2004) opina que nos hallamos ante un texto científico, cuya «función predominante es sin duda la referencial, puesto que se tratan de textos expositivos-explicativos [...]».

De esta diversidad en la catalogación de diferentes textos, particularmente en el ámbito especializado, se desprende que las diversas tipologías textuales pueden suponer un obstáculo a la hora de extraer las características prototípicas de estos textos ya que, de acuerdo con las diferentes tipologías, se basará en unos $\mathrm{u}$ otros aspectos del propio texto, en este caso, del prospecto. Para evitar esta problemática, se han publicado diversos estudios a nivel nacional e internacional con el fin de realizar una aproximación al prospecto como género desde una perspectiva tanto lingüística (Barrio Cantalejo, Simón-Lordà, Melguizo, Escalona, Marijuán y Hernando, 2008; Ballesteros Peña y Fernández Aedo, 2013) como traductológica (Mercado López, 2004; Blancafort Alias, de Cambra Florensa y Navarro Rubio, 2006; García Izquierdo, 2008; Ruiz Garrido, Fortanet Gómez y Palmer Silveira, 2008; Vázquez y del Árbol, 2013, 2014).

Estas investigaciones indican una clara tendencia al estudio de aspectos concretos como la legibilidad y comprensión del prospecto de me- 
406 dicamentos para el consumo humano, para lo que se cuentan con instrumentos como el índice de Flesch-Szigriszt y la escala Inflesz (Ballesteros y Fernández, 2013). Por otra parte, se puede observar la evidente tendencia a realizar estudios contrastivos para la extracción de rasgos prototípicos macroestructurales y léxicos generalmente entre el par de lenguas inglés-español, lo que resalta una gran ausencia de otras combinaciones lingüísticas y de problemáticas directamente relacionadas con las características lingüísticas propias de combinaciones lingüísticas diferentes (Rodríguez Martínez, 2017).

De este hecho se desprende la necesidad de llevar a cabo investigaciones que contemplen aspectos que, por las características intrínsecas de la lengua inglesa, no se tienen en cuenta como las dificultades asociadas a la variación morfológica entre lenguas flexivas afines como es el francés y el español, cuya cercanía sintáctica puede llegar a producir errores al traspasar el contenido especializado a la lengua meta. Por otro lado, y debido a los trabajos que se han realizado desde múltiples perspectivas tanto lingüísticas y traductológicas (contenido, legibilidad, estructura, legislación, etc.) sobre el prospecto de medicamentos para uso humano, hacen que este género textual presente una literatura científica sobre la que continuar estos estudios en otras combinaciones lingüísticas y poder así contrastar las dificultades específicas asociadas a la lengua en la traducción biosanitaria.

\section{METODOLOGÍA}

Con objeto de demostrar la presencia de variación morfológica en el discurso médico francésespañol, se ha realizado un corpus de prospectos de medicamentos comercializados en Francia y en España de diversos principios activos con el fin de extraer y clasificar casos de variación de rasgos morfológicos.
El tipo de texto, el prospecto médico, se ha seleccionado por ser un género sujeto a una terminología estandarizada y estructuras reguladas por normativas nacionales e internacionales, tales como las plantillas QRD propuestas por la EMA, los Standard Terms, el MedDRA y otros recursos multilingües creados por instituciones europeas, así como por contar con una alta densidad terminológica (Mayor Serrano, 2009). Por otro lado, los numerosos estudios que han perfilado el género textual del prospecto en la combinación lingüística inglés-español proporcionan información sobre los aspectos lingüísticos del prospecto en español que podrían guiar las investigaciones en otras lenguas menos estudiadas, como es el caso del francés.

Los prospectos que componen el corpus del presente estudio fueron seleccionados ajustando las limitaciones diasistemáticas a prospectos comercializados en Francia (para el subcorpus en francés) y en España (para el subcorpus en español). Para ello, la compilación del corpus se llevó a cabo con prospectos oficiales en francés, descargados de la Agence nationale de sécurité $d u$ médicament et des produits de santé (ANSM) ${ }^{1}$, y en español, descargados del Centro de Información online de Medicamentos de la AEMPS (CIMA) ${ }^{2}$.

Para la discriminación de textos en la base de datos, se decidió seguir los criterios de estudios anteriores sobre prospectos realizados con corpus. Así, los requisitos para la selección textual fue los principios activos de diferentes grupos farmacológicos de mayor consumo en envases. Con este fin, se revisaron los informes oficiales sobre el sector farmacéutico y sanitario en Francia (ANSM, 2014) y en España (Ministerio de Sanidad, Servicios Sociales e Igualdad, 2015). Tras analizar las ventas de medicamentos en ambos

I Disponible en https://ansm.sante.fr/.

2 Disponible en https://cima.aemps.es/cima/publico/ home.html. 
países, los principios activos y sus correspondientes subgrupos farmacológicos ATC4 que se seleccionaron para el corpus fueron los siguientes: omeprazol (A02BC; antiulceroso: inhibidor de la bomba de protones), atorvastatina (C10AA; hipolipemiante: inhibidor de la HMG CoA reductasa), ácido acetilsalicílico (B01AC; inhibidor de la agregación plaquetaria, excluyendo heparina), diclofenaco (M01AE; derivado del ácido propiónico), paroxetina (NO6AB; antidepresivo: inhibidor selectivo de la recaptación de serotonina), tramadol-paracetamol (NO2AX; analgésico), furosemida (CO3CA; diuréticos de techo alto: sulfonamidas, monofármacos) y amoxicilina (J01CA; antiinfeccioso: antibacteriano be- talactámico, penicilinas). Gracias a la legislación 407 europea vigente, que obliga a tener una copia digital del prospecto de todo medicamento comercializado en la UE, se ha podido compilar una muestra totalmente homogénea en términos de cantidad y de correspondencia textual. Así, se han recopilado 10 prospectos de cada uno de los siete principios activos (ácido acetilsalicílico, amoxicilina, atorvastatina, diclofenaco, furosemida, omeprazol y paroxetina) y del compuesto medicamentoso (tramadol-paracetamol) por idioma, lo que generaría un corpus de 160 textos.

Las características principales del corpus del presente estudio se recogen en la siguiente tabla (véase Tabla 1):

Tabla 1. Características del corpus de prospectos de medicamentos para uso humano (francés-español)

\begin{tabular}{|c|c|}
\hline Textos compilados & 80 (TOFRA) y 80 (TOESP) \\
\hline Palabras (tokens) & 380595 \\
\hline Formas (types) & 9443 \\
\hline Ratio Type/Token & $2,48 \%$ \\
\hline Género textual & Prospecto de medicamentos para uso humano \\
\hline Temática & Biosanitaria, farmacéutica \\
\hline Idioma & Francés y español \\
\hline Restricciones diatópicas & Francés europeo y español peninsular \\
\hline Originalidad del texto & Original \\
\hline Tamaño del texto & Texto completo \\
\hline Documentado & Sí \\
\hline Etiquetado & Sí \\
\hline Anotaciones & No \\
\hline
\end{tabular}


408 Como se desprende del número de palabras proporcionadas en la Tabla 1, el corpus compilado es un corpus comparable ad hoc. En este tipo de corpus, la cantidad de palabras no suele contemplarse como una característica prioritaria, sino que es la calidad de los textos lo que posee una mayor relevancia (Corpas Pastor, 2001), avalada dicha calidad en nuestro caso por la descarga de los prospectos oficiales mediante las plataformas gubernamenta- les de Francia (ANSM) y de España (CIMA).

$\mathrm{Al}$ tratarse de un género textual breve, conciso y con una variación sintáctica y léxica muy baja, la representatividad del corpus se puede alcanzar con un número reducido de textos, tal y como se observa en la siguiente ilustración (véase Figura 1), realizada con el programa ReCor (creado por Gloria Corpas Pastor, Miriam Seghiri Domínguez y Romano Maggi, Universidad de Málaga):
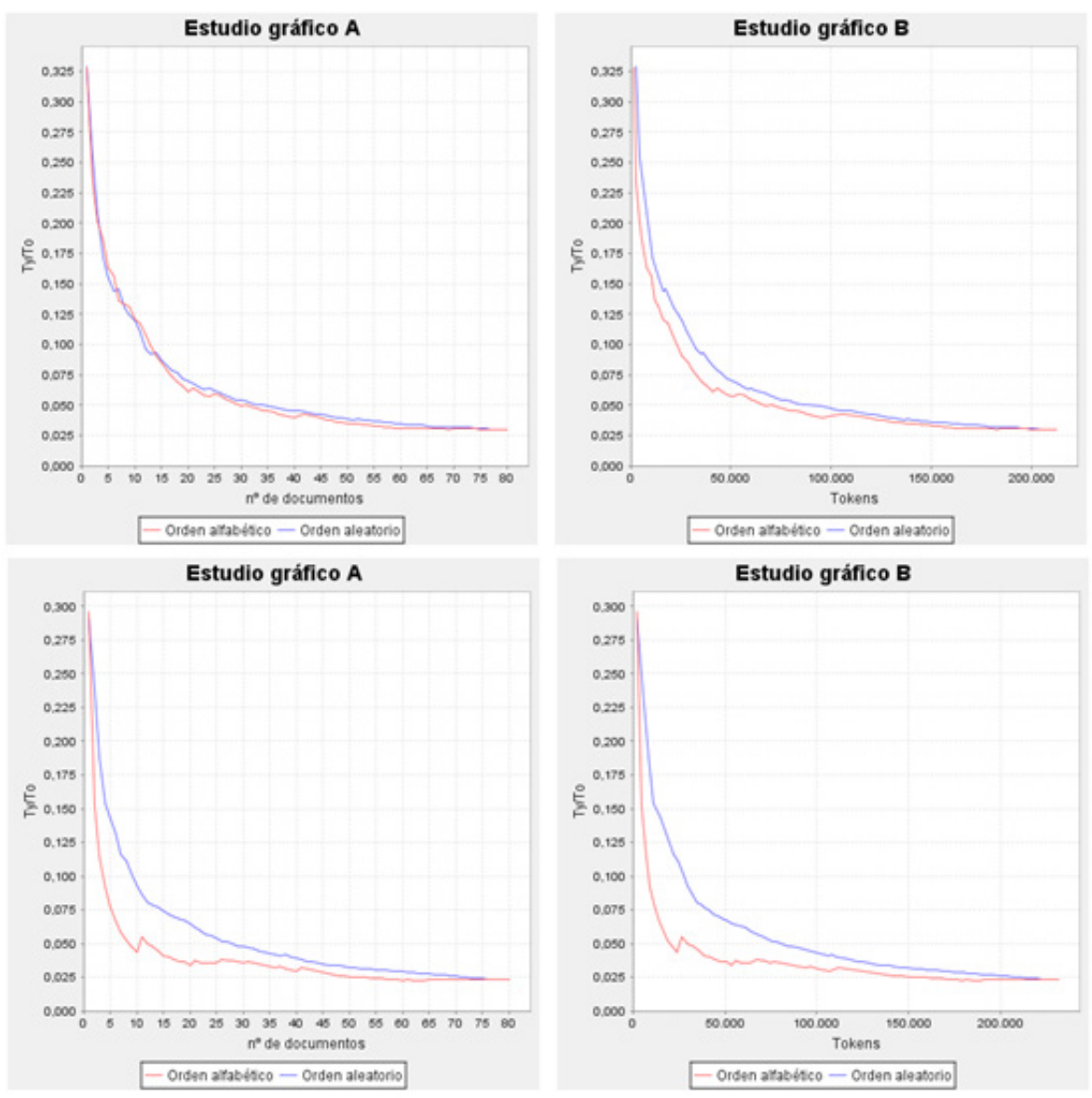

FIGURA I. Representatividad del corpus de prospectos de medicamentos para uso humano en español (arriba) y francés (abajo) a través de ReCor, programa creado por Gloria Corpas Pastor, Miriam Seghiri Domínguezy Romano Maggi Domínguez (Universidad de Málaga) 
Tal y como se observa en esta ilustración, la representatividad de nuestro corpus en español se alcanzó a partir de 60 documentos, o lo que es lo mismo, a partir de aproximadamente 175000 palabras; por otro lado, la representatividad de nuestro corpus en francés se alcanzó a partir de 72 documentos o a partir de 200000 palabras. A partir de la cantidad de textos y palabras citadas, la muestra no incorpora una cantidad de formas nuevas relevantes (la línea se estabiliza en un punto cercano al cero), lo cual indicaría que la muestra se considerara representativa.

\section{Herramienta empleada}

Las herramientas de gestión de corpus proporcionan unas funcionalidades similares. No obstante, para el presente estudio hemos utilizado la herramienta en línea Sketch Engine ${ }^{3}$. Es una herramienta que debido a su versatilidad, posee gran potencial para la práctica profesional y para la investigación. Se ha seleccionado esta herramienta por su calidad de herramienta en línea, lo que permite una mayor facilidad para acceder a la información y para el almacenamiento del corpus, así como por su herramienta de etiquetación automática POS Tagger. Gracias a esta herramienta, se ha realizado una anotación lingüística de manera automática, lo que facilita la recuperación de información en Sketch Engine al poder llevar a cabo búsquedas mediante CQL y expresiones regulares.

\section{VARIACIÓN MORFOLÓGICA}

Tras compilar el corpus, se ha realizado una selección y catalogación de los ejemplos de casos con variación morfológica entre el francés y el español que presentan una frecuencia relevante en el corpus analizado.

\footnotetext{
3 Disponible en https://www.sketchengine.eu/.
}

Como mencionábamos anteriormente, un gran número de investigadores abordan el estudio de la traducción médica desde un punto de vista descriptivo de corte terminológico partiendo del inglés como lengua de partida. La investigación actual suele centrarse en la identificación y estudio de rasgos morfosintácticos (Sevilla Muñoz, 2004; Ruiz Rosendo, 2007, López Garrido, 2016; Rodríguez Ponce, 2017), lo que acaba relegando a un segundo plano las variaciones morfológicas en lenguas con flexión de género y número como es el caso del francés y del español, que podríamos achacar a la influencia del inglés como lingua franca (Navarro, 2001, 2006), en beneficio de los rasgos sintácticos como, por ejemplo, la voz pasiva (Navarro et al., 1997) o el gerundio (Mendiluce Cabrera, 2002).

Por este motivo, en este artículo nos proponemos realizar una aproximación a las particularidades a nivel morfológico presentes en el discurso médico entre la combinación lingüística del francés hacia el español a través del prospecto de medicamentos para uso humano como objeto de análisis. Así, se podrán analizar las variaciones morfológicas entre dichos idiomas para ayudar así a su ilustrar este problema de traducción específico dentro del ámbito de la traducción biosanitaria, ya que, como menciona Sánchez Trigo (2002: 697), estas dificultades morfológicas se originan «por las diferencias entre las lenguas de trabajo más que por problemas específicos de traducción».

A continuación, proponemos una clasificación de los casos más relevantes identificados a lo largo del análisis de corpus, que se organizarán como variación de número y variación de género.

\section{Variación de número}

Como miembros de diferentes comunidades lingüísticas (francófonos e hispanohablantes), existen diferencias en las convenciones que ri- 
gen, entre otras muchas, el uso del plural y el singular. Según Martínez López (2010b: 400), «el francés prefiere el plural para designar, de forma genérica, a enfermedades que pueden afectar a uno o más órganos o que presentan una o varias modalidades de aparición. El español, por su parte, prefiere el singular».

Así, la autora propone los siguientes ejemplos para ilustrar esta realidad, a los que hemos añadido otros ejemplos extraídos de la práctica profesional de los autores del presente artículo:

$$
\begin{aligned}
& \text { brûlure des mains }>\text { quemaduras de la mano } \\
& \text { syndromes coronariens }>\text { síndrome coronario } \\
& \text { maladies des pieds }>\text { enfermedades del pie } \\
& \text { examen clinique des seins }>\text { exploración mamaria } \\
& \text { analyse de cheveux }>\text { análisis capilar }
\end{aligned}
$$

Por otro lado, Martínez López (2010b: 400) afirma también que el francés suele usar el plural para aludir a un conjunto, mientras que el español tiende a emplear el singular:

$$
\begin{aligned}
& \text { renseignements > información } \\
& \text { sorties d'eau > salida de agua } \\
& \text { matières fécales > materia fecal }
\end{aligned}
$$

Los casos que menciona la autora, y que exponemos anteriormente, se repiten en el corpus objeto de estudio, tal y como mostramos a continuación. En la siguiente tabla (véase Tabla 2) se observa una tendencia por parte del francés a pluralizar al existir diferentes modalidades de aparición de enfermedades o enfermedades que afectan diferentes órganos, así como otras unidades léxicas que recurren al mismo patrón morfológico.

Si bien es cierto que estas equivalencias también se pueden presentar en plural, suelen aparecer con una frecuencia bastante menor, lo cual confirma la teoría que exponemos al comienzo de este apartado. Este es el caso de la construcción dificultad para + verbo, que tras una búsqueda lematizada en nuestro corpus, recuperamos un total de 207 resultados, en los cuales 184 están redactadas en singular y únicamente 23 en plural. Cabe mencionar también que de los 23 resultados de la construcción en plural, 10 de ellos se corresponden con una frase que se repite en todos los prospectos de medicamentos con omeprazol, y otros 10 de ellos con otra frase que se repite en todos los prospectos de medicamentos con el compuesto medicamentoso tramadol-paraceta-

Tabla 2. Diferencias de uso del número entre francés y español en el lenguaje médico: plural francés y singular español

\begin{tabular}{cccc}
\hline Francés & Frec. & Español & Frec. \\
\hline dans les urines & 35 & en la orina & 12 \\
\hline difficultés à (respirer, avaler, uriner) & 113 & dificultad para (tragar, respirar, orinar) & 184 \\
\hline douleurs (articulaires, abdominales) & 535 & dolor (articular, abdominal) & 733 \\
\hline Saignements & 203 & sangrado/hemorragia & $73 / 186$ \\
\hline maux de (tête, gorge, dos, estomac) & 136 & $\begin{array}{c}\text { dolor de (cabeza, garganta, espalda, estóma- } \\
\text { go)/dolor (abdominal, estomacal) }\end{array}$ & 249 \\
\hline ordures ménagères & 79 & basura & 79 \\
\hline informations importantes & 54 & información adicional & 150 \\
\hline
\end{tabular}


mol. De esta manera, vemos que a pesar de tener una frecuencia de 23 en nuestro corpus, esta frecuencia se debe a la formación de nuestro corpus y a la repetición de estructuras en contenidos similares. Esto mismo sucede con el caso de la construcción dolor + adjetivo o dolor de + sustantivo, cuya frecuencia de uso nos indica una clara tendencia al uso en singular (675) frente al uso en plural (58). Igualmente, es de especial interés observar cómo estas frecuencias de uso se aglutinan en prospectos de unos determinados principios activos, omeprazol, amoxicilina y el compuesto medicamentoso tramadol-paracetamol.

No obstante, a lo largo del corpus encontramos también casos en los que el francés emplea el singular mientras que el español emplea el plural, tal y como aparece en la siguiente tabla.

Al igual que en la Tabla 2, en esta tabla se muestran las equivalencias que se han localizado como prioritarias en términos de frecuencia. Por ejemplo, la búsqueda lematizada de la construcción nivel de + sustantivo nos recupera unos 259 casos, 236 casos en los que está expresada en plural y solamente 23 casos en los que está expresada en singular. En la misma línea que lo expuesto anteriormente, los casos con menor frecuencia de uso se suelen concentrar en prospectos con el mismo principio activo, como es el caso de nivel de potasio (localizado en 7 prospec- tos de furosemida) o niveles de azúcar (localizado en 7 prospectos de ácido acetilsalicílico).

Otro dato que se desprende de la tabla es que, si bien la forma alteración de también sería una propuesta idiomática y válida para altération de, en español suele presentar una frecuencia mucho menor (17) frente a la forma en plural alteraciones de (85).

Es también de especial interés el término contraceptif (que da lugar a la construcción méthode contraceptive), que se ha expresado en los prospectos españoles como anticonceptivo (medidas anticonceptivas). Si bien es cierto que el DLE recoge la voz contraceptivo como correcta, al igual que la forma mixta contraconceptivo, las fuentes de consulta especializadas recomiendan el uso de anticonceptivo (Navarro, 2020).

De estas tablas (véanse Tabla 2 y Tabla 3 ) se desprende que la combinación lingüística francés-español requiere una especial atención a la variación de número, dado que la misma procedencia de familias lingüísticas puede ejercer una gran influencia a la hora de tomar decisiones de traducción (Sánchez Trigo, 2002). En este caso, se deduce que el traductor de textos de temática biosanitaria entre el francés y el español habría de prestar especial atención a estos casos en los que, debido a diferentes fenómenos de evolución lingüística o rasgos morfológicos, los

Tabla 3. Diferencias de uso del número entre francés y español en el lenguaje médico: singular francés y plural español

\begin{tabular}{|c|c|c|c|}
\hline Francés & Frec. & Español & Frec. \\
\hline crise (d'épilepsie, d'asthme, cardiaque) & 55 & $\begin{array}{c}\text { crisis (epilépticas, cardíacas, convulsivas, } \\
\text { hipertensivas) }\end{array}$ & 43 \\
\hline taux de (sucre, lipide, cholestérol) & 204 & niveles de (azúcar, lípidos, colesterol) & 236 \\
\hline méthode contraceptive & 1 & medidas anticonceptivas & 20 \\
\hline altération de & 10 & alteraciones de & 85 \\
\hline
\end{tabular}


412 usos del plural y del singular difieren en cuanto a la frecuencia de uso. Esto es particularmente relevante en el caso del francés y el español ya que, debido a esta cercanía léxica, sintáctica, semántica y estilística, se pueden producir calcos de diversa índole (estructural, estilístico, léxico, etc.) (Ortega Arjonilla y Echeverría Pereda, 1996), que el traductor debería evitar mediante el conocimiento de estos fenómenos y de todas las implicaciones subyacentes, así como de una documentación exhaustiva realizada con numerosos recursos (no solamente generales) para evitar estos problemas específicos de variaciones de número (Quérin, 2017).

\section{Variación de género}

A diferencia del inglés, el francés y el español son lenguas que poseen género gramatical. Este hecho influye directamente en la traducción bio- sanitaria puesto que, según Echeverría Pereda y Jiménez Gutiérrez (2010), y dado que las terminologías especializadas aspiran a ser unívocas, la correspondencia entre los diferentes equivalentes de un término en varias lenguas habría de ser total en todos los aspectos. Sin embargo, observamos que las autoras muestran en su artículo variaciones de género dentro de la Terminologia Anatomica (TA) entre el francés y el español:

processus alvéolaire (masc.) > apófisis alveolar (fem.)

sillon olfactif (masc.) $>$ fosa olfatoria (fem.) gouttière lacrymale (fem.) > surco lagrimal (masc.) arcade zygomatique (fem.) $>$ arco zigomático (masc.)

Estas variaciones hacen que el grado de precisión de la terminología médica se vea mermado (Echeverría Pereda y Jiménez Gutiérrez, 2010),

Tabla 4. Variaciones de género gramatical entre francés y español en el lenguaje médico: femenino francés y masculino español

\begin{tabular}{cccc}
\hline Francés & Frec. & Español & Frec. \\
\hline analyse (fem.) & 56 & análisis (masc.) & 92 \\
\hline attaque (fem.) & 49 & ataque (masc.) & 85 \\
\hline douleur (fem.) & 535 & dolor (masc.) & 733 \\
\hline greffe (fem.) & 13 & transplante/trasplante (masc.) & $8 / 21$ \\
\hline humeur (fem.) & 66 & humor (masc.) & 35 \\
\hline méthode contraceptive (fem.) & 1 & método anticonceptivo (masc.) & 3 \\
\hline oedème (fem.) & 91 & edema (masc.) & 48 \\
\hline oreille (fem.) & 76 & oreja (fem.)/oído (masc.) & $0 / 105$ \\
\hline pensée (fem.) & 43 & pensamientos (masc.) & 89 \\
\hline poudre (fem.) & 195 & polvo (masc.) & 79 \\
\hline rougeur (fem.) & 54 & enrojecimiento (masc.) & 37 \\
\hline substance active (fem.) & 190 & principio activo (masc.) & 103 \\
\hline
\end{tabular}


Tabla 5. Variaciones de género gramatical entre francés y español en el lenguaje médico: masculino francés y femenino español

\begin{tabular}{cccc}
\hline Francés & Frec. & Español & Frec. \\
\hline arrêt (masc.) & 143 & interrupción (fem.) & 23 \\
\hline asthme (masc.) & 85 & asma (fem.) & 77 \\
\hline cellulose (masc.) & 29 & celulosa (fem.) & 29 \\
\hline gonflement (masc.) & 174 & hinchazón (fem.) & 138 \\
\hline lactose (masc.) & 35 & lactosa (fem.) & 50 \\
\hline saignement (masc.) & 203 & hemorragia (fem.) & 186 \\
\hline sang (masc.) & 501 & sangre (fem.) & 636 \\
\hline ulcère (masc.) & 225 & úlcera (fem). & 243 \\
\hline
\end{tabular}

dado que se podrían producir variaciones de género en determinantes y complementos que acompañan a estos términos. Estas variaciones de género se extienden a todos los ámbitos de la medicina, no solo a la anatomía, como vemos en los siguientes ejemplos extraídos de la práctica profesional de los autores del presente artículo:

$$
\begin{aligned}
& \text { échantillon (masc.) > muestra (fem.) } \\
& \text { don d'ovules (masc.) > donación de óvulos (fem.) } \\
& \text { notice }(\text { fem.) }>\text { prospecto (masc.) } \\
& \text { poudre (fem.) > polvo (masc.) }
\end{aligned}
$$

Tal y como sucede en el caso de la variación de número que exponemos en el apartado anterior, el análisis de corpus nos devuelve resultados que confirman este fenómeno en el lenguaje médico entre el francés y el español. Variaciones de género gramatical entre francés y español en el lenguaje médico: masculino francés y femenino español.

En las tablas anteriores (véanse Tabla 4 y Tabla 5) se muestran casos de variación de género en el lenguaje médico entre francés y español. Sin embargo, en el género textual que nos ocupa, el prospecto de medicamentos para uso humano, encontramos variaciones de género también de términos del lenguaje corriente, como es el caso de lait (masc.) > leche (fem.).

En nuestro caso son especialmente relevantes los casos que podrían resultar en una propuesta de traducción poco idiomática o, tal y como demuestran las frecuencias de uso de nuestro corpus, en propuestas que no se corresponden con lo más empleado en este género textual. Así, observamos el caso de méthode contraceptive (1) que, como especificábamos anteriormente en el apartado de variación de número, la equivalencia más frecuente la presenta medidas anticonceptivas (20). No obstante, encontramos también el equivalente métodos anticonceptivos, con una frecuencia de uso muy inferior (3), la cual se localiza únicamente en un prospecto de medicamentos de ácido acetilsalicílico, mientras que medidas anticonceptivas se encuentra localizada de manera homogénea en todos los prospectos de atorvastatina. Estas propuestas poco extendidas y con una frecuencia mucho menor que las empleadas en el género textual se pueden deber a interferencias bidireccionales que se dan 
414 en lenguas afines y que generan soluciones automatizadas (Cagnolati, 2015), principalmente ante una construcción que tendría sentido traspasar de manera literal en la lengua meta.

De igual modo, el caso de substance active (190) puede dar lugar mediante estas interferencias la propuesta sustancia activa (7) en lugar de generar la propuesta más idiomática, principio activo (103) por influencia del género del texto origen. Como en la mayoría de los casos presentados, este tipo de errores se suelen propagar en el corpus gracias a los prospectos del mismo medicamento, en este caso, de furosemida.

El caso de saignement (203) nos muestra otra potencial interferencia, que por influencia del género y la forma original se trasvase como sangrado (73), cuando la propuesta más acertada en español según fuentes especializadas (Navarro, 2020) y la frecuencia de uso (186) es hemorragia.

En este mismo sentido, tenemos el caso de oreille, que se puede traducir por oreja (0), más influenciado por el texto origen, o por oído (105), una propuesta más correcta según el contexto en el que nos encontramos el término: sonnerie dans les oreilles $>$ zumbidos en los oídos.

Además, y tal como afirmaba Quérin (2013), encontramos términos que llegan a alternar géneros, como es el caso de enzyme > enzima:
Estos casos demuestran el problema de traducción que implica este fenómeno. Si bien es cierto que la adquisición de una competencia lingüística alta puede ayudar al traductor a identificar los cambios de género gramatical de términos no especializados (como el caso de lait > leche), la práctica profesional y los recursos con información contextual son de vital importancia para garantizar la total identificación del género gramatical de estas unidades especializadas, lo que evitaría asíla redacción de frases con incoherencias no solo gramaticales y sintácticas, sino también morfológicas, que garanticen una mayor idiomaticidad. A pesar de que los recursos disponibles para la traducción médica pueden resultar de apoyo para la práctica profesional, los diccionarios especializados suelen proporcionar poca información en este sentido (Rouleau, 2001) e implica una necesidad de documentación contextual para el traductor especializado en el ámbito biosanitario.

\section{CONCLUSIONES}

Tal y como apuntábamos durante el diseño metodológico del artículo, el prospecto de medicamentos para uso humano se convierte en un género textual óptimo para la investigación en

Tabla 6. Variaciones de género gramatical entre francés y español en el lenguaje médico: diferentes contextos de uso de ambos géneros

\begin{tabular}{|c|c|}
\hline Francés & Español \\
\hline [...] (manque en un enzyme des globules rouges). & $\begin{array}{c}{[\ldots] \text { aumento moderado de las enzimas hepáticas }} \\
{[\ldots]}\end{array}$ \\
\hline $\begin{array}{l}\text { les inhibiteurs de l'enzyme de conversion, les antago- } \\
\text { nistes du récepteur à l'angiotensine II, le méthotrexate } \\
\text { utilisé à des doses inférieures à } 20 \mathrm{mg} / \mathrm{semaine}[. . .]\end{array}$ & $\begin{array}{c}{[. . .] \text { si está siendo tratado con medicamentos }} \\
\text { denominados inhibidores del enzima monoamino } \\
\text { oxidasa }[. . .]\end{array}$ \\
\hline
\end{tabular}


terminología biosanitaria debido a la brevedad de este género textual, así como a su densidad terminológica. Además, dada la tendencia de estandarización terminológica a la que está sometido desde instituciones nacionales e internacionales, lo convierte en un género textual que requiere de bastante menos muestra para lograr compilar un corpus representativo (60-72 textos/175 000-200 000 palabras).

Gracias a la representatividad del corpus, hemos podido observar que las variaciones morfológicas en la combinación lingüística francés-español se dan con cierta frecuencia en el lenguaje de especialidad biosanitario.

Las variaciones de número suelen deberse a motivos culturales, como es la identificación de un término que representa un conjunto con uno $\mathrm{u}$ otro número, por lo que puede llegar a ser más fácil de identificar y de solventar como problema de traducción. Sin embargo, la variación de género no suele atender a ninguna convención o norma, por lo que estas variaciones aleatorias plantean un problema de traducción mayor y podrían provocar una traducción de textos de contenido biosanitario con errores morfológicos. Si bien es cierto que estas propuestas se deben a interferencias por afinidad sintáctica y morfológica, estas alteraciones se podrían paliar con una formación adecuada que contemplase estas particularidades morfológicas y una extensa práctica profesional.

A este respecto, los corpus se perfilan como una herramienta versátil y complementaria no solo a la labor investigadora sino también a la práctica profesional con la que localizar contextos de uso. Además, con el uso de CQL y expresiones regulares se puede recuperar información en una sola búsqueda, cuyos resultados contemplen la variación morfológica. Una vez lematizado el corpus con el etiquetado automático de estas herramientas, dicha búsqueda puede filtrar- se por lemas y establecer una equivalencia con información cuantitativa (frecuencias de uso) y cualitativa (contextos de uso especializados). De esta manera, el corpus al servicio de los traductores médicos con combinaciones lingüísticas que no contemplen el inglés como lengua de trabajo, y por consiguiente tengan una menor cantidad de recursos terminológicos y documentales a su disposición, podrían realizar búsquedas complejas con las que localizar equivalentes contextualizados y conocimiento especializado. A pesar de que este tipo de búsquedas requiere cierta práctica con la sintaxis de CQL y expresiones regulares, su incorporación al flujo de trabajo traductor e investigador genera unos resultados más precisos, más contextualizados y con menos ruido informativo.

\section{REFERENCIAS BIBLIOGRÁFICAS}

Alarcos Llorach, Emilio (2009): Gramática de la Lengua Española, Madrid: Espasa.

ANSM (2014): Analyse des ventes de médicaments en France en 2013 [en línea], París: ANSM <http:// ansm.sante.fr/var/ansm_site/storage/original/ap plication/3df7b99f8f4c9ee634a6a9b094624341. pdf $>$ [consulta: 05-VII-2020].

Ballesteros Peña, Sendoa e Irrintzi Fernández Aedo (2013): «Análisis de la legibilidad lingüística de los prospectos de los medicamentos mediante el índice de Flesch-Szigriszt y la escala Inflesz», Anales del Sistema Sanitario de Navarra, 36 (3), 397-406.

Barrio Cantalejo, Inés María; Pablo Simón-Lordá; Miguel Melguizo; Isabel Escalona; María Isabel MARIJUÁn y Pablo Hernando (2008): «Legibilidad gramatical de los prospectos de los medicamentos de más consumo y facturación en España en 2005», Anales del Sistema Sanitario de Navarra, 31 (2), 559 566.

Blancafort Alias, Sergi; Salomé de Cambra Florensa y M. a Dolors NAVArro Rubio (coords.) (2006): Funciones y utilidad del prospecto del medicamento, Barcelona: Fundació Biblioteca Josep Laporta. 
416

Cagnolati, Beatriz Emilce (2015): «Interferencia en la traducción francés-español de textos de ciencias sociales», Hikma, 14, 55-74.

CoRpas PASTOR, Gloria (2001): «Compilación de un corpus ad hoc para la enseñanza de la traducción inversa especializada», TRANS, 5, 155-184.

Crespo Hidalgo, Juan (2010): «Estudio cienciométrico de las tesis doctorales sobre Traducción e Interpretación en España (1972-2010)», en Emilio ORTEGA Arjonilla, Ana Belén Martínez López y Elena EcheveRRíA Pereda (eds.), Panorama actual de la investigación en traducción e interpretación, Granada: Atrio, 35-92.

Díaz Alarcón, Soledad (2016): «Introducción a la traducción médica francés-español: los folletos de salud»,Panace@17 (43), 4-15.

Durán-Escribano, Pilar e Irina ArgüElles-Álvarez (2017): «Domain-specific mappings as conceptual categorisers for scientific and technical vocabularies», Ibérica, 34, 137-162.

Echeverría Pereda, Elena e Isabel JimÉnez Gutiérrez (2010): «La terminología anatómica en español, inglés y francés», Panace@, 11 (31), 47-57.

Eurrutia Cavero, Mercedes (2017): «La formación de palabras en francés médico y su incidencia en el proceso de traducción de textos médicos del francés al español», en Emilio Ortega Arjonilla y Ana Belén MARTínez López (eds.), Traducción e Interpretación en el ámbito biosanitario, Granada: Comares, 545-562.

Gamero Pérez, Silvia (1999-2000): «La traducción de textos técnicos y la diversidad tipológica», Sendebar, 10-11, 127-158.

García Izquierdo, Juan Manuel (2008). «Estudio descriptivo del prospecto de medicamentos desde una perspectiva traductológica». En Miguel Ángel Campos Pardillos y Adelina Gómez González-Jover (eds.): The Language of Health Care: Proceedings of the first International Conference on Language and Health Care, Alicante, 24-26 de octubre, 2007.

López GARRIDo, Salomé de los Ángeles (2016): La neología por préstamo en el discurso médico: análisis de los procedimientos de creación léxica y de las estrategias de traducción, Tesis doctoral, Universidad de Alicante.

López Rodríguez, Clara Inés (2000): «Tipologías textuales y géneros en la normalización terminoló- gica y ortotipográfica de la traducción médica», Terminologie et traduction, 3, 95-115.

Martínez López, Ana Belén (2008): La traducción editorial de manuales especializados dentro del ámbito biosanitario: Aplicaciones a la enseñanza y a la práctica profesional de la traducción médica del inglés al español, Tesis doctoral, Universidad de Granada.

Martínez López, Ana Belén (2009): «Sobre la traducción de documentos médico-legales (de español a inglés): práctica profesional y explotación didáctica en el aula de traducción especializada», Redit: Revista electrónica de didáctica de la traducción y la interpretación, 2, 33-52.

Martínez López, Ana Belén (2010a): «La terminología médica en francés, inglés y español: problemas que se derivan de la presencia del inglés como lingua franca de la comunicación científica a escala internacional», Anales de Filología Francesa, 18, 393-404.

Martínez López, Ana Belén (2010b): «La investigación en traducción médica: estado de la cuestión», en Emilio Ortega Arjonilla, Ana Belén Martínez López y Elena Echeverría Pereda (eds.), Panorama actual de la investigación en traducción e interpretación, Granada: Atrio, 1059-1084.

Martínez Motos, Raquel (2015): «El género prospecto y el efecto de la traducción inglés-español en su legibilidad y facilidad de uso para el lector lego», Tesis doctoral, Universidad de Alicante.

Mayor Serrano, M. ${ }^{\text {a }}$ Blanca (2007): «La importancia de la tipología textual pragmática para la formación de traductores médicos»,Panace@, 9 (26),124-137.

MAYOR SERrano, Blanca (2009): «Recomendaciones para la elaboración de los prospectos de los medicamentos»,Panace@, 10 (29),103-104.

Mendiluce Cabrera, Gustavo (2002): «El gerundio médico»,Panace@, 3 (7),74-78.

Mercado López, Sebastián (2004). «El análisis de géneros aplicado a la traducción: Los prospectos de medicamentos de Estados Unidos y España», Linguax: Revista de lenguas aplicadas, 2 , sin paginación.

Ministerio de Sanidad, Servicios Sociales e Igualdad (2015). Informe anual del Sistema Nacional de Salud 2015 [en línea], Madrid: Ministerio de Sanidad, Servicios Sociales e Igualdad, Observatorio del Sistema Nacional de Salud. <https://www.msssi. gob.es/estadEstudios/estadisticas/sisInfSanSNS/ 
tablasEstadisticas/Inf_Anual_SNS_2015.1.pdf> [consulta: 05-VII-2020].

MuÑoz-Miquel, Anna (2009): «El acceso al campo profesional de la traducción médica: hacia una definición social del traductor médico», Fòrum de Recerca, 14, 312-329.

Muñoz-Miquel, Anna (2014): «El perfil y las competencias del traductor médico desde el punto de vista de los profesionales: una aproximación cualitativa»,Panace@, 30 (10),157-167.

MuÑoz Torres, Carlos Arturo (2002): «Tipología textual y análisis para la traducción. Una tipología de géneros médicos», en José CHABÁS, Rolf GASER y Joëlle REY VANIN (eds.), Translating Science. Proceedings $2^{\text {nd }}$ International Conference on Specialized Translation, Barcelona: Universitat Pompeu Fabra, 319-325.

NAVARro, Fernando (2001): «El inglés, idioma internacional de la medicina»,Panace@ 3 (2), 35-51.

NAVARro, Fernando (2006): «La anglización del español: mucho más allá de bypass, piercing, test, airbag, container y spa», en Luis GonZÁlez y Pollux HernúÑeZ (coords.), Traducción: Contacto y contagio. Actas del III Congreso Internacional El Español, lengua de traducción. Puebla (México), Bruselas: ESLEtRA, 231232.

NAVArro, Fernando (2020): Libro Rojo. Diccionario de dudas y dificultades de traducción del inglés médico (3. a edición). [en línea] <https://www.cosnautas. com/es> [consulta: 03-VII-2020].

NAVARro, Fernando y Francisco Hernández (1997): «Anatomía de la traducción médica», en Emilio Ortega Arjonilla y Leandro Félix Fernández (coords.), Lecciones de teoría y práctica de la traducción, Málaga: Universidad de Málaga, Servicio de Publicaciones, 137-162.

Navarro, Fernando, Francisco Hernández y Lydia RoDRíguez-Villanueva (1997): «Uso y abuso de la voz pasiva en el lenguaje médico escrito», en Fernando NAVARro (coord.), Traducción y lenguaje en medicina, Barcelona: Fundación Dr. Antoni Esteve, 101-106.

Ortega Arjonilla, Emilio (2003): «Aspectos metodológicos de la traducción científica y técnica. Aplicaciones al ámbito francés-español», en Miguel Ángel García Peinado y Emilio Ortega Arjonilla (coords.), Panorama actual de la investigación en traducción e interpretación, Granada: Atrio, 199-234.

Ortega Arjonilla, Emilio y Elena Echeverría Pereda (1996): Enseñanza de Lenguas, Traducción e Interpretación (Francés-Español), Málaga: Servicio de Publicaciones y Divulgación Científica de la Universidad de Málaga.

Ortega Arjonilla, Emilio y Ana Belén Martínez López (2007): «La terminología médica en clave traductológica: convencionalismo, normalización, redundancia y reproductibilidad», Sendebar, 18, 263-288.

PÉrez García, Encarnación (2004): «Los prospectos: estudio de lo tecnolectal hacia lo divulgativo», Revista Electrónica de Estudios Filológicos, 8.

QuÉrIN, Serge (2013): «Variation terminologique en français médical», Panace@, 38 (14), 235-239.

QuérIN, Serge (2017): «Pluriel caché dans les noms composés en anglais médical», Traduire, 236, 13-19.

Rodríguez Martínez, Manuel Cristóbal (2017): «Particularidades de la traducción en el ámbito biosanitario del francés al español», en Emilio ORTEGA Arjonilla, Ana Belén Martínez López y Francisca GARcía Luque (eds.), Cartografía de la traducción, la interpretación y las industrias de la lengua. Mundo profesional y formación académica: interrogantes y desafíos, Granada: Comares, 151-166.

Rodríguez Martínez, Manuel Cristóbal y Emilio Ortega Arjonilla (2018): «El corpus de prospectos farmacéuticos como recurso didáctico en el aula de traducción especializada francés-español», MonTI, 10, 117-140.

Rodríguez Ponce, María Isabel (2017): «Aspectos morfosintácticos y léxicos en la traducción al español de How to do things with words», Onomázein, 38, 1-21.

Rouleau, Maurice (2001): «La facture des principaux dictionnaires médicaux français : point de vue d'un traducteur», Meta: Journal des traducteurs / Meta: Translator's Journal, 46 (1), 34-55.

Ruiz Garrido, Miguel; Inmaculada Fortanet Gómez y Juan Carlos Palmer SiLveira (2008). «El prospecto de medicamentos en España y EEUU: utilización de viñetas y abreviaturas». En Miguel Ángel Campos Pardillos y Adelina Gómez González-Jover 
418 (eds.): The Language of Health Care: Proceedings of the first International Conference on Language and Health Care, Alicante, 24-26 de octubre, 2007.

Ruiz Rosendo, Lucía (2007): «El predominio del inglés en el lenguaje científico: características del lenguaje médico español en la actualidad», Polissema, 7, 85-113.

SÁnchez Trigo, Elena (2002): «La traducción de textos médicos del francés al español», en Maria Carme Figuerola Cabrol, Montse Parra y Pere Solà (eds.), La lingüistica francesa en el nuevo milenio, Lérida: Milenio, 689-698.

SÁnchez Trigo, Elena y Laura Munoa (2013): «Lengua francesa y traducción en el ámbito biomédico y científico»,Panace@, 14 (38),171-175.

Sánchez Trigo, Elena y Tamara Varela Vila (2015): «Traducción de referencias culturales en textos biomédicos sobre enfermedades neuromusculares (francés-español)», Çédille: revista de estudios franceses, 11, 501-528.

Sevilla Muñoz, Manuel (2004): «Hacia la definición de necesidades de aprendizaje de los alumnos de Traducción Científico-Técnica», Panace@, 5 (16), 141-148.

VÁzquez y del Árbol, Esther (2013). «Traditional patient information leaflet frente al prospecto medicamentoso tradicional: el orden de los factores ¿no altera? el valor del producto», Panace@, 14 (37), 89-97.

VÁzQuez y del ÁRBol, Esther (2014). «Modern user information leaflet frente al prospecto medicamentoso: el orden de los factores ¿no altera? el valor del producto (segunda parte)»,Panace@, 15 (39), 118-127.

Veloso, Isabel (2004): «Ortotipografía comparada (francés-español)», Thélème. Revista Complutense de Estudios Franceses, 19. 183-194. 